\title{
Manejo quirúrgico y no quirúrgico de la osteoartritis en el atleta. Una revisión sistemática de estudios recientes
}

\author{
Surgical and non-surgical management of osteoarthritis in the \\ athlete. A systematic review of recent studies
}

\author{
Félix Enrique Villalobos Córdova, ${ }^{*}$ Francisco Cruz López, * Gerardo Meraz Lares, \\ Emmanuel Díaz de León Miranda, § Fernando Rangel Gutiérrez," José Luis Martínez Hernández," \\ Santiago Alfaro Barbosa, ${ }^{* *}$ Carlos Alberto Calzada Chong, ${ }^{\neq \neq}$Jairo José Ferniza Garza, $\$ \S$ \\ Carlos Andrés Navarrete Sarasty, 97 Jorge Romo Rivera***
}

*Médico adscrito al Servicio de Ortopedia del Deporte y Artroscopia, Instituto Nacional de Rehabilitación "Luis Guillermo Ibarra Ibarra»; ${ }^{*}$ Médico Especialista en Ortopedia, Hospital Médica Sur. Jefe de Servicios Médicos de Cruz Azul FC; $§$ Jefe de los Servicios Médicos del Atlético de San Luis. Director Médico del CAR La Loma, San Luis Potosí; "Médico adscrito al Servicio de Artroscopia, Unidad Médica de Atención Ambulatoria No. 90, IMSS, Torreón, Coahuila; "Médico Especialista en Rehabilitación, Director Médico de Sport Care SA; **Profesor en la Universidad Autónoma de Guadalajara. Asesor Médico de la LIGA CUFAO de Flag Football; ¥¥Médico adscrito al Centro de Traumatología Deportiva y Ortopedia, Sports Clinic Tampico, Tamaulipas; \&\$ Médico residente de Alta Especialidad en Cirugía Articular, Instituto Nacional de Rehabilitación "Luis Guillermo Ibarra Ibarra"; "19Médico residente de Alta Especialidad en Artroscopia y Lesiones Deportivas, Instituto Nacional de Rehabilitación “Luis Guillermo Ibarra Ibarra»; ;**Médico adscrito en Traumatología del Deporte, Hospital Ángeles Lomas.

\section{Resumen}

Introducción: Las lesiones condrales son hallazgos comunes en la cirugía artroscópica, pudiendo progresar a osteoartritis de manera temprana en pacientes deportistas, causando limitación funcional en población económicamente activa. El objetivo de este estudio fue realizar una revisión sistemática y análisis cualitativo de las terapias posibles para pacientes jóvenes y deportistas con osteoartritis. Métodos: Se realizó una revisión sistemática en las bases de datos de Medline, PubMed y Cochrane, con términos de búsqueda divididos en subgrupos para osteoartritis de rodilla, hombro, tobillo, tratamiento farmacológico y rehabilitación física; con filtro de búsqueda a estudios de mayor evidencia clínica publicados en los últimos cinco años. Resultados: Se obtuvieron un total de 1,078 registros, descartando 821 al revisar los resúmenes, con 257 artículos en texto completo revisados y 69 seleccionados para realizar el análisis cualitativo. Conclusiones: En los pacientes deportistas que sufren lesiones articulares, se cuenta con varias opciones de tratamiento para que puedan retomar su práctica deportiva o al menos disminuir su progresión a osteoartritis; sin embargo, algunos tratamientos aún no tienen respaldo de evidencia clínica para recomendarlas.

Palabras clave: Osteoartritis, manejo quirúrgico, manejo no quirúrgico, atletas.

Nivel de evidencia: III.

\section{Abstract}

Introduction: Chondral lesions are common findings in arthroscopic surgery, and can progress to early osteoarthritis in sports patients, causing functional limitation in the economically active population. The aim of this study was to carry out a systematic review and qualitative analysis of possible therapies for young patients and athletes with osteoarthritis. Methods: A systematic review was carried out in the Medline, PubMed and Cochrane databases, with search terms divided into subgroups for osteoarthritis of the knee, shoulder, ankle, pharmacological treatment and physical rehabilitation; with a search filter to studies with the most clinical evidence published in the last 5 years. Results: A total of 1,078 records were obtained, discarding 821 when reviewing the abstracts, with 257 full-text articles reviewed and 69 selected for qualitative analysis. Conclusions: In sports patients who suffer joint injuries, there are several treatment options so

\section{Correspondencia:}

Dr. Félix Enrique Villalobos Córdova

Ave. Mayorazgo No. 130, consultorio 704, Col. Xoco, 03330,

Alcaldía Benito Juárez, Ciudad de México. Tel: 55 5601-6965,

E-mail: villalobosenrique@ hotmail.com. josemajimeneza@tec.mx
Recibido: 24-01-2021. Aceptado: 08-02-2021.

Citar como: Villalobos CFE, Cruz LF, Meraz LG, Díaz de León ME, Rangel GF, Martínez HJL, et al. Manejo quirúrgico y no quirúrgico de la osteoartritis en el atleta. Una revisión sistemática de estudios recientes. Orthotips. 2021; 17 (1): 32-42. https://dx.doi.org/10.35366/99165 
that they can resume their sports practice or at least reduce their progression to osteoarthritis, however, some treatments still do not have clinical evidence support to recommend them.

Keywords: Osteoarthritis, surgical management, non-surgical management, athletes.

Level of evidence: III.

\section{Introducción}

Las lesiones condrales son hallazgos comunes en la cirugía de artroscopia, reportadas hasta en $60 \% \cdot^{1-3}$ El daño por lesión ligamentaria, lesión condral o fractura en una articulación desencadena apoptosis de condrocitos, hemartrosis y reacción sinovial que evolucionan con el tiempo a ruptura de la matriz extracelular y degradación del cartílago articular, ${ }^{4}$ siguiendo así una historia natural de lesión articular hacia la osteoartritis en un periodo de $15-20$ años, ${ }^{5}$ dando como resultado adultos jóvenes, muchos de ellos deportistas, económicamente activos y con necesidad de trabajar, que ya tienen una limitación o discapacidad funcional en alguna o varias de sus articulaciones principales.

Existen factores de riesgo descritos para la osteoartritis, tanto sistémicos como la edad, sexo, raza, densidad mineral ósea, hormonas sexuales, nutricionales y genéticos, así como factores locales o biomecánicos como la obesidad, daño articular, práctica del deporte y debilidad muscular. ${ }^{6}$

En los atletas, la osteoartritis resulta de una interacción de factores biológicos, mecánicos y biomecánicos, complicados por lesiones traumáticas previas que aceleran los procesos patológicos a nivel de sus articulaciones. En la actualidad, existen ya publicadas guías de práctica clínica para el diagnóstico y manejo de la osteoartritis (AAOS, OARSI, Guías Mexicanas), pero no existen en nuestro conocimiento revisiones sistemáticas que respondan sobre el manejo de la osteoartritis en el atleta. Consideramos que en los últimos años las opciones de manejo para la osteoartritis han evolucionado en forma rápida, dando así nuevas opciones de tratamiento para enriquecer el manejo multimodal de la osteoartritis en el deportista.

El presente trabajo tiene como objetivo contestar las siguientes preguntas:

1. ¿Cuáles son los tratamientos y resultados para el manejo quirúrgico de la osteoartritis en rodilla, tobillo y hombro en el atleta publicados entre 2013 y 2018 ?
2. ¿Cuáles son los tratamientos y resultados para el manejo no quirúrgico de la osteoartritis en el atleta publicados entre 2013 y 2018 ?

3. ¿Cuáles son los tratamientos y resultados para el manejo con rehabilitación de la osteoartritis en el atleta publicados entre 2013 y 2018 ?

\section{Métodos}

Protocolo: la metodología de búsqueda y criterios de inclusión de los estudios se estipuló en un protocolo registrado en el International Register of Systematic Reviews (PROSPERO, número de registro: 114297).

Criterios de elección: ensayos clínicos aleatorizados, metaanálisis, revisiones sistemáticas, guías de práctica clínica y ensayos clínicos controlados, estudios en humanos. Sin restricción de lenguaje, con fechas de publicación en los últimos cinco años (de octubre 2013 a octubre 2018).

Tipo de intervención: estudios en atletas adultos con lesiones grado I a III de osteoartritis de rodilla, tobillo u hombro, incluyendo tratamientos no quirúrgicos y quirúrgicos. Se excluyeron estudios en adolescentes (menores de 18 años) o gente mayor de 70 años, además de aquéllos con lesiones grado IV de osteoartritis.

Tipo de resultados: cualquiera que demuestre o no una diferencia o recomendación para seguir algún tipo de terapia, fármaco o intervención quirúrgica.

Fuentes de información: se realizó la búsqueda en bases de datos electrónicas, artículos en otros idiomas fueron traducidos. Las bases de datos utilizadas fueron PubMed, Medline y The Cocharne Library. La última búsqueda fue hecha el día 29 de septiembre de 2018.

Estrategia de búsqueda: se realizó dividida en subgrupos, usando un conjunto de términos para cada uno (Tabla 1). Con contención de búsqueda a ensayos clínicos controlados, metaanálisis, estudios multicéntricos, guía de práctica clínica, ensayo clínico controlado aleatorizado y revisiones sistemáticas, estudios en humanos, publicados entre octubre 2013 hasta octubre 2018. 
Selección de estudios: la valoración de los estudios para su elección fue realizada por dos autores, sin aplicación de ciego.

Proceso de recolección de resultados: dos revisores independientes fueron los encargados de seleccionar los artículos que cumplían con los criterios de inclusión, con un tercer revisor independiente en caso de existir controversias, y después fueron analizados utilizando el flujograma PRISMA para determinar sesgos y sintetizados mediante narrativa. En el caso de estudios relacionados específicamente con tratamientos para la osteoartritis, se clasificaron por dos revisores independientes y un tercer revisor en caso de existir controversias, con base en los niveles de recomendación propuestos por Shekelle y colaboradores (Tabla 2).

ITEM de datos: la información fue extraída de los estudios de acuerdo con: 1) características de los participantes (edad, sitio de lesión); 2) tipo de intervención (tratamiento quirúrgico y/o no quirúrgico); y 3 ) resultado medido (escalas funcionales, dolor, diferencias clínicas).

Riesgo de sesgo en estudios individuales: para validar el riesgo de sesgo de las publicaciones se valoró secuencia de encubrimiento, cegado de participantes y/o tratantes, pacientes perdidos en seguimiento, seguimiento de la intención de tratar, falta de datos y otros sesgos.

\section{Resultados}

La selección y características de los estudios se muestran en la Tabla 1.

\section{Síntesis de estudios}

Tratamiento quirúrgico de la osteoartritis de rodilla en el atleta: en una revisión sistemática con metaanálisis en pacientes con lesión de ligamento cruzado anterior se reportó un riesgo relativo $(\mathrm{RR})$ de 3.89 para desarrollar osteoartritis menor y 3.84 para osteoartritis moderada a severa. Los casos con lesión de ligamento cruzado anterior tienen un RR mayor de desarrollar cualquier grado de osteoartritis comparado con aquéllos tratados con cirugía de reconstrucción (4.98 vs 3.62). La progresión a osteoartritis moderada o severa (grado III o IV) después de 10 años en casos con reconstrucción de ligamento cruzado anterior es mayor comparado con los manejados sin cirugía (4.71 vs 2.41), esto quizá se debe a que los pacientes

Tabla 1: Flujograma de PRISMA.

\begin{tabular}{|c|c|c|c|c|c|}
\hline Item/subgrupo & $\begin{array}{l}\text { Osteoartritis de } \\
\text { rodilla }\end{array}$ & $\begin{array}{l}\text { Osteoartritis de } \\
\text { hombro }\end{array}$ & $\begin{array}{l}\text { Osteoartritis de } \\
\text { tobillo }\end{array}$ & Tratamiento farmacológico & Rehabilitación \\
\hline Términos de búsqueda & $\begin{array}{l}\text { Osteoarthritis and } \\
\text { sports and knee }\end{array}$ & $\begin{array}{l}\text { Osteoarthritis } \\
\text { and sport and } \\
\text { shoulder }\end{array}$ & $\begin{array}{l}\text { Osteoarthritis and } \\
\text { sport and ankle }\end{array}$ & $\begin{array}{l}\text { Osteoarthritis and knee and } \\
\text { (viscosupplementation OR corticosteroids } \\
\text { OR platelet rich plasma OR stem cells) }\end{array}$ & $\begin{array}{c}\text { Osteoarthritis and } \\
\text { sport and rehabilitation }\end{array}$ \\
\hline $\begin{array}{l}\text { Características de } \\
\text { búsqueda }\end{array}$ & \multicolumn{5}{|c|}{$\begin{array}{l}\text { Se establecieron criterios de búsqueda para ensayos clínicos controlados, metaanálisis, estudios multicéntricos, guía de } \\
\text { práctica clínica, ensayo clínico controlado aleatorizado y revisiones sistemáticas, estudios en humanos, publicados entre } \\
\text { octubre } 2013 \text { hasta octubre } 2018 \text {. }\end{array}$} \\
\hline Registros identificados & 535 & 35 & 32 & 185 & 291 \\
\hline \multicolumn{6}{|l|}{ Revisión de resúmenes } \\
\hline Descartados & 443 & 20 & 19 & 65 & 183 \\
\hline Seleccionados & 92 & 15 & 13 & 120 & 108 \\
\hline $\begin{array}{l}\text { Artículos en texto } \\
\text { completo revisados }\end{array}$ & 92 & 15 & 13 & 119 & 18 \\
\hline $\begin{array}{l}\text { Estudios seleccionados } \\
\text { para síntesis cualitativa }\end{array}$ & 7 & 2 & 6 & 44 & 11 \\
\hline $\begin{array}{l}\text { Características de } \\
\text { selección }\end{array}$ & $\begin{array}{l}1 \text { metaanálisis } \\
3 \text { revisiones } \\
\text { sistemáticas } \\
1 \text { ensayo clínico } \\
\text { aleatorizado } \\
1 \text { guía clínica } \\
1 \text { serie de casos } \\
\text { multicéntrica }\end{array}$ & $\begin{array}{l}1 \text { metaanálisis } \\
1 \text { revisión } \\
\text { sistemática }\end{array}$ & $\begin{array}{l}5 \text { revisiones } \\
\text { sistemáticas } \\
1 \text { ensayo clínico } \\
\text { aleatorizado }\end{array}$ & $\begin{array}{c}8 \text { metaanálisis } \\
8 \text { revisiones sistemáticas } \\
1 \text { ensayo clínico controlado } \\
19 \text { ensayos clínicos aleatorizados } \\
5 \text { guías clínicas } \\
1 \text { serie de casos } \\
1 \text { estudio de cohorte prospectivo } \\
1 \text { estudio quasi-experimental }\end{array}$ & $\begin{array}{l}5 \text { metaanálisis } \\
3 \text { revisiones } \\
\text { sistemáticas } \\
\quad 3 \text { guías }\end{array}$ \\
\hline
\end{tabular}


Tabla 2: Clasificación de la información.

Categoría de la evidencia

la. Evidencia de metaanálisis de los estudios clínicos aleatorios

lb. Evidencia de por lo menos un estudio clínico controlado aleatorio

Ila. Evidencia de por lo menos un estudio controlado sin aleatoriedad

Ilb. Al menos otro tipo de estudio cuasiexperimental o estudios de cohorte

III. Evidencia de un estudio descriptivo no experimental, tal como estudios comparativos, estudios de correlación, casos y controles y revisiones clínicas

IV. Evidencia de comité de expertos, reportes, opiniones o experiencias clínicas de autoridades en la materia o ambas. Artículo de revisión clínica
Fuerza de la recomendación

A. Directamente basad en evidencia categoría I

B. Directamente basada en evidencia categoría II o recomendaciones extrapoladas de evidencia I

C. Directamente basada en evidencia categoría III o en recomendaciones extrapoladas de evidencias categorías I o II

D. Directamente basadas en evidencia categoría IV o de recomendaciones extrapoladas de evidencias categorías II, III

Modificado de: Shekelle P, et al. 1999.?

operados regresan en mayor proporción a actividades de riesgo, a comparación de los no operados. ${ }^{8}$

En una revisión sistemática de estudios longitudinales de resonancia magnética nuclear posterior a la reconstrucción de ligamento cruzado anterior, Van Ginckel y colaboradores observaron la presencia de osteoartritis desde los dos años de seguimiento tras la lesión inicial. Los cambios morfológicos en el primer año fueron agravados por la reconstrucción de ligamento cruzado anterior en comparación con los que fueron tratados sin cirugía. ${ }^{9}$

En un ensayo clínico controlado aleatorizado, Tsoukas y su equipo asignaron 17 de 32 pacientes a manejo quirúrgico de reconstrucción de ligamento cruzado anterior con autoinjerto de isquiotibiales, contra 15 de manejo no quirúrgico, con un seguimiento promedio de 10 años, encontrando mejores resultados funcionales y menor laxitud en el grupo de operados, pero la incidencia de cambios radiológicos de osteoartritis fue similar. ${ }^{10}$

En un estudio multicéntrico, Gomoll y su grupo de investigadores recomiendan la técnica de implante de condrocitos autólogos en adultos jóvenes con lesiones condrales de espesor total de patela grado III-IV del International Cartilage Repair Society (ICRS), debiendo tratar también alteraciones biomecánicas concomitantes. ${ }^{11}$

En una revisión sistemática realizada por Paul Monk y colaboradores en 2016 se encontró evidencia de alto nivel de que la meniscectomía no es superior al manejo no quirúrgico como estrategia de primera línea en pacientes con gonalgia y desgarro meniscal degenerativo. Alguna evidencia refiere que los casos con síntomas persistentes que hayan fallado a manejo no quirúrgico se pueden beneficiar del desbridamiento meniscal como segunda línea de tratamiento. ${ }^{12}$

En un Consenso realizado por la European Society of Sports Traumatology, Knee Surgery, Arthroscopy (ESSKA) publicado en 2017 establecen que la artroscopia con meniscectomía parcial debe considerarse después de una evaluación estandarizada clínica y radiográfica, cuando la respuesta al manejo no quirúrgico no haya sido satisfactoria por al menos tres meses, y sin datos de osteoartritis radiográfica y con lesión meniscal grado III en resonancia magnética. ${ }^{13}$

Con base en una revisión sistemática de HongWei Chen y colaboradores, se recomienda que al realizar tratamientos de osteosíntesis en las fracturas de meseta tibial se realice asistencia con artroscopia para tener un menor daño a tejidos y una adecuada visualización de la reducción del espacio articular. ${ }^{14}$

Tratamiento quirúrgico de la osteoartritis de hombro en el atleta: en una revisión sistemática de estudios nivel IV Namdari y colaboradores ${ }^{15}$ refieren mejora en dolor y satisfacción del paciente en el corto plazo con el uso del desbridamiento artroscópico para el tratamiento de la artritis glenohumeral. En esta publicación establecen que falta evidencia de alta calidad que soporte el uso de este tratamiento.

En una revisión sistemática con meta-análisis, Sayegh y su equipo ${ }^{16}$ encontraron que la artroplastia total de hombro da un mayor control de dolor y rango de movilidad que lo que da la hemiartroplastia en el tratamiento de pacientes jóvenes con artritis glenohumeral. Consideran el desbridamiento artroscópico como una alternativa eficaz y segura en el corto plazo para sujetos jóvenes que no desean artroplastia.

Tratamiento quirúrgico de la osteoartritis de tobillo en el atleta: en una revisión sistemática sobre el ma- 
nejo de la osteoartritis de tobillo, Bloch y colaboradores $^{17}$ reportan mayor efectividad del desbridamiento artroscópico en el pinzamiento anterior moderado y artritis temprana, que en la artritis avanzada difusa en tobillo. La artroplastia de distracción se indica en osteoartritis en etapa final en pacientes jóvenes con alineación cercana a la normal, que prefieren retrasar la artrodesis o la artroplastia definitiva. La osteotomía de realineación supramaleolar está indicada en casos con mala alineación, artritis localizada, secundario a fracturas o en pacientes jóvenes que no desean artrodesis 0 artroplastia, con resultados generalmente buenos hasta por ocho años, pero con porcentajes altos de revisión. El uso de aloinjertos osteocondrales también está reportado para casos con osteoartritis de tobillo en etapa final con mínima mala alineación, con tibia distal y astrágalo bien vascularizado, pero con evidencia insuficiente para sugerir su uso. Finalmente hablan de la artrodesis de tobillo como el tratamiento estándar de la osteoartritis de tobillo.

En una revisión sistemática, Noailles y su equipo ${ }^{18}$ revisaron el manejo quirúrgico de la inestabilidad de tobillo con reparación anatómica directa versus reparación no anatómica. Encontrando mejores resultados funcionales y menos osteoartritis secundaria con la reparación anatómica directa del ligamento peroneo-astragalino anterior y peroneo-calcáneo. Las técnicas no anatómicas pueden causar osteoartritis secundaria y pérdida en el rango de movilidad del tobillo, por lo que no las recomiendan como primera línea de tratamiento.

Ross y colaboradores ${ }^{19}$ hicieron una revisión del síndrome de pinzamiento anterior de tobillo y comentan que el tratamiento abierto es efectivo pero asociado a un número importante de complicaciones, considerando a la artroscopia anterior de tobillo como un abordaje efectivo en el tratamiento del pinzamiento óseo o de partes blandas y con 3.5\% de complicaciones reportadas y con un regreso a la actividad deportiva en siete semanas.

En un ensayo clínico controlado aleatorizado, Cadossi y su grupo de investigadores ${ }^{20}$ reportan el uso de concentrado de células de médula ósea, con estimulación biofísica postquirúrgica (lo que protege a las células trasplantadas del efecto catabólico de citocinas inflamatorias), presentando mejoría en cuestionario de AOFAS y menor dolor en comparación al uso de las células de médula ósea sin la estimulación biofísica, a más de un año de cirugía.

En una revisión sistemática publicada por VanTienderen y colaboradores ${ }^{21}$ sobre el uso de aloinjertos osteocondrales para el tratamiento de lesiones osteocondrales grandes en astrágalo (OCLT, por sus siglas en inglés), encontraron mejora en el cuestionario AOFAS y mejora del dolor en EVA en el postoperatorio, $25 \%$ de tasa de reoperación y $8.8 \%$ que acabaron en artrodesis o reemplazo de tobillo. Los autores sugieren usar modalidades de tratamiento menos invasivas y con menor morbilidad antes de considerar el uso de un implante de aloinjerto osteocondral.

En otra revisión sistemática de Johnson y su equipo, ${ }^{22}$ sobre el uso de aloinjertos osteocondrales en tobillo, en 15 artículos analizados reportan una incidencia de complicaciones asociadas de 13 hasta 42\%; sin embargo, lo consideran una opción favorable para el tratamiento de pacientes con osteoartritis de tobillo de estadio final.

Tratamiento farmacológico de la osteoartritis en el atleta:

\section{Viscosuplementación}

Se recomienda el uso de viscosuplementación en el manejo de la osteoartritis en el deportista, en dos revisiones sistemáticas, un ensayo clínico controlado, dos estudios multicéntricos, ${ }^{23-29}$ siendo mejor que la infiltración de esteroides, ${ }^{30}$ igual que terapia física ${ }^{31}$ e infiltrar plasma rico en plaquetas, ${ }^{32}$ infiltración con polinucleótidos, ${ }^{33}$ infiltrar ácido hialurónico combinado con Manitol y sin que se produzcan cambios estructurales en el cartílago detectables por resonancia magnética nuclear con gadolinio, ${ }^{34}$ pero sí disminución de biomarcadores séricos de osteoartritis. ${ }^{35}$ No se recomienda la viscosuplementación en el manejo de la osteoartritis inmediatamente después de cirugía de meniscectomía $a^{36}$ o de reconstrucción de ligamento cruzado anterior. ${ }^{37}$ En dos artículos, un metaanálisis ${ }^{38}$ y la Guía de Práctica Clínica del American Academy of Orthopaedic Surgeons (AAOS), ${ }^{39}$ ambos del mismo autor Jevsevar, no recomiendan el uso de viscosuplementación en osteoartritis de rodilla. Finalmente en una revisión sistemática ${ }^{40}$ y un análisis de guías de práctica clínica ${ }^{41}$ los resultados no son concluyentes.

\section{Células madre mesenquimales}

Lamo-Espinosa y colaboradores ${ }^{42}$ en un ensayo clínico aleatorizado de células madre mesenquimales de médula ósea autóloga de 30 pacientes aleatorizados en tres grupos (ácido hialurónico, baja dosis 
de células madre, alta dosis de células madre) en pacientes de edades entre 55 y 61 años (para los grupos control y de alta dosis, con 10 pacientes cada uno), obtuvieron resultados de mejoría en 16 puntos en la escala de WOMAC a 12 meses de seguimiento, además de mejoría en la EVA y en la reducción del espacio articular.

Shapiro y su grupo ${ }^{43}$ en un ensayo clínico aleatorizado de infiltración en rodilla con concentrado de aspiración de médula ósea vs solución salina no demostraron diferencias significativas entre ambos grupos, aunque refieren mejoría en EVA y mediciones de la Sociedad Internacional de Investigación de Osteoartritis (OARSI) a seis meses de seguimiento en ambos grupos.

Xia y su equipo ${ }^{44}$ en un metaanálisis de siete ensayos clínicos con 314 pacientes en total reportan una leve mejoría en cuanto a dolor y función física de los pacientes tratados con aspirados de médula ósea, además de ser seguro; sin embargo, debido a la alta heterogeneidad de los estudios y su pobre poder metodológico, no justifican su recomendación.

Yubo y colaboradores ${ }^{45}$ en un metaanálisis de 11 ensayos clínicos con 582 pacientes demuestran una mejoría en la valoración WOMAC y Lequesne para el grupo experimental con células madre, siendo seguro, pero con el inconveniente de que sólo la mitad de los estudios estaban cegados, hubo alta heterogeneidad, las escalas son subjetivas y el estudio es multicéntrico, por lo que sólo se establece como potencialmente benéfico.

Pas y su grupo de investigadores ${ }^{46}$ en una revisión sistemática, detectan que el principal inconveniente de los ensayos clínicos de células madre es su alto contenido de sesgos, además de la diferencia entre obtención de las células madre, por lo que no recomiendan su uso hasta tener ensayos clínicos de mayor fuerza metodológica.

\section{Plasma rico en plaquetas (PRP)}

En una revisión sistemática realizada por Meheux y colaboradores ${ }^{47}$ de ensayos clínicos nivel I se concluye que en casos con osteoartritis de rodilla sintomática la infiltración de PRP resulta en mejoría clínica hasta por 12 meses tras la administración, siendo mejores los resultados clínicos y la valoración WOMAC en comparación con ácido hialurónico.

Dai y su grupo, ${ }^{48}$ en un metaanálisis de 1,069 pacientes en 10 ensayos clínicos aleatorizados de PRP vs hialuronato, refieren que hay mejoría en la escala del dolor WOMAC y escala funcional WOMAC con significancia estadística en el grupo PRP a los 12 meses tras la intervención comparado con el grupo control.

Laudy y su equipo, ${ }^{49}$ en un metaanálisis de 10 ensayos clínicos con 1,110 casos, obtuvieron un resultado en favor del PRP con mejoría en la reducción del dolor y en la EVA comparado con hialuronato y placebo. Aunque la mayoría de los ensayos clínicos tenían alto riesgo de sesgo y una heterogeneidad considerable.

Smith y colaboradores, ${ }^{50}$ en un ensayo clínico doble ciego vs placebo de 30 pacientes (15:15), indican que no hubo reacciones adversas del PRP en el grupo experimental, y que hay una mejoría de $78 \%$ en la evaluación WOMAC comparada con $7 \%$ en el grupo placebo.

Simental y su grupo de investigadores, ${ }^{51}$ en un ensayo clínico aleatorizado de PRP bajo en leucocitos vs acetaminofén en 65 pacientes (no cegado) con gonartrosis grado I-II, demostraron superioridad del grupo de PRP al mejorar el QOL, disminución del dolor y mejoría de la funcionalidad de la rodilla, atribuido principalmente a las citocinas antiinflamatorias que presenta el PRP.

Gormeli y colaboradores, ${ }^{52}$ en un ensayo clínico aleatorizado doble ciego con 162 pacientes dividido en 4 grupos (múltiples administraciones de PRP, administración simple de PRP, hialuronato y placebo), concluyeron que en casos de estadios de osteoartritis I-III la administración de PRP seriado tiene mejores resultados que el ácido hialurónico y la dosis simple de PRP, y éstos a su vez superioridad a placebo. En estadios grado IV no presenta beneficio alguno.

Gobbi y su equipo de investigadores, ${ }^{53}$ en un ensayo clínico aleatorizado no cegado de 119 rodillas en pacientes activos deportivamente, con estadio de gonartrosis I-II e IMC menor de 30, fueron aleatorizados entre un grupo de PRP simple y otro de PRP en tres dosis (una aplicación por mes), por un seguimiento de dos años. En ambos grupos hubo mejoría comparado con los datos base, pero en el grupo de administración múltiple tuvo aún mejores resultados a los 12 meses, los cuales pueden ser prolongados al repetir el ciclo hasta dos años, después de los cuales no se observa mejoría e incluso decremento.

Filardo y colegas, ${ }^{32}$ en un ensayo clínico aleatorizado no cegado de 143 pacientes con estadio de gonartrosis de I-III, compararon PRP vs viscosuplementación sin encontrar diferencia significativa alguna entre escalas clínicas y de dolor. 
Cole y colaboradores, ${ }^{54}$ en un ensayo clínico aleatorizado doble ciego con 111 pacientes entre los grupos de PRP y ácido hialurónico, concluyeron que en las valoraciones subjetivas los pacientes en el grupo de PRP tendían a tener mejores resultados; sin embargo, en las valoraciones objetivas no se encontró diferencia.

\section{Glucosamina, condroitina y otros suplementos}

Se recomienda el uso de glucosamina y condroitina de acuerdo a Zeng ${ }^{55}$ en pacientes con gonartrosis grado $>2$ de Lawrence, ya que los pacientes presentan mejoría clínica tras el tratamiento con ellos, y además una disminución del estrechamiento articular, aunque leve, sin mostrar superioridad vs celecoxib.

Un ensayo clínico aleatorizado refiere mejoría de la locomoción y la fuerza cuadricipital tras 16 y ocho semanas respectivamente de tratamiento con glucosamina/condroitina/péptido de colágeno. ${ }^{56}$

Kongtharvonskul y colaboradores en un metaanálisis ${ }^{57}$ concluyen que el tratamiento con glucosamina tiene mejoría clínica en las evaluaciones WOMAC y EVA comparado con placebo; sin embargo, no modifica la evolución de la enfermedad en cuanto a pérdida de espacio articular.

La ESCEO ${ }^{58}$ dentro de su esquema de manejo de la osteoartritis presenta la glucosamina como parte del tratamiento base en pacientes sintomáticos. La OARS ${ }^{59}$ concluye una indicación incierta para su uso en mejoría de la sintomatología, pero no recomendada como modificador de enfermedad.

Por otra parte, no se recomienda el uso de glucosamina, condroitina $y / u$ otros suplementos basados en dos ensayos clínicos y un metaanálisis, ya que no se logró determinar si presenta alguna mejoría tanto clínica ${ }^{60}$ como en las evaluaciones de cartílago por imagenología ${ }^{61}$ con el uso de glucosamina y otros suplementos; mientras que el metaanálisis ${ }^{62}$ no demostró diferencia entre glucosamina y placebo.

\section{Esteroides}

Jüni y colaboradores, ${ }^{63}$ en un metaanálisis de 26 ensayos clínicos controlados de corticosteroides intraarticulares vs control, determinaron que el tratamiento con corticosteroides da una mejoría clínica significativa las primeras seis semanas, tras las cuales se pierde el efecto.
La Sociedad Internacional de Investigación de Osteoartritis (OARSI) dentro de sus guías de recomendaciones, encabezadas por McAlindon y colaboradores, ${ }^{59}$ estipula a los esteroides tanto en osteoartritis sin comorbilidades como en aquella con comorbilidades, teniendo mejores resultados a corto plazo que el ácido hialurónico.

Soriano-Maldonado y su grupo, ${ }^{64}$ en un ensayo clínico aleatorizado de 100 pacientes de metilprednisolona/lidocaína vs solución salina como terapia de apoyo dos semanas antes de un periodo de ejercicio de 12 semanas y valorados mediante umbrales de tolerancia al dolor, no demostraron diferencia significativa entre ambos grupos durante el régimen de actividades.

Popma y su equipo, ${ }^{65}$ en un ensayo clínico de 97 pacientes comparando 40 vs $80 \mathrm{mg}$ de triamcinolona de manera intraarticular en osteoartritis de rodilla, no demostraron ventaja alguna de duplicar la dosis en los pacientes.

McAlindon y colaboradores, ${ }^{66}$ en un ensayo clínico aleatorizado de 140 pacientes de osteoartritis de rodilla grado 2-3 tratados con infiltraciones con triamcinolona vs placebo (solución salina) en ciclos de 12 semanas por dos años, demostraron mayor pérdida de volumen de cartílago en el grupo experimental, sin diferencia de sintomatología al término del seguimiento.

Tratamiento de rehabilitacion en la osteoatritis en el atleta:

\section{Terapia física}

Las guías actuales muestran que la rehabilitación y fisioterapia disminuyen el dolor, aumentan la fuerza muscular y mejoran la funcionalidad en casos con osteoartritis leve a moderada al aplicar ejercicio terapéutico, el ejercicio neuromotor, la terapia manual y la educación. ${ }^{38,59,67}$ De acuerdo con las recomendaciones de OARSI de 2014, la rehabilitación se considera el tratamiento principal de la osteoartritis y se recomienda para todos los pacientes. ${ }^{59}$

\section{Fortalecimiento muscular}

Un metaanálisis reciente apoya la evidencia previa de los beneficios del ejercicio en el tratamiento de la osteoartritis, los ejercicios que aumentan la fuerza, la flexibilidad y la capacidad aeróbica probablemente son los más efectivos en osteoartritis de miembro inferior. ${ }^{68}$ 
La Guía de OARSI en un metaanálisis y revisión sistemática demostró moderado efecto del entrenamiento de fortalecimiento muscular para disminuir el dolor y mejorar la función física en osteoartritis de rodilla y multiarticular con o sin comorbilidades. ${ }^{59}$

En un ensayo clínico controlado aleatorizado con 100 pacientes con osteoartritis de rodilla y severa disfunción física se encontró que por cada Nw/kg que se incremente la fuerza de los extensores de rodilla se asocia con una mejoría en la función física de 17 unidades WOMAC. ${ }^{69}$

Un metaanálisis y revisión sistemática demostró disminución moderada del dolor y mejoría de la funcionalidad con entrenamiento de fuerza en comparación con el grupo control, concluyendo que el fortalecimiento muscular isocinético es una forma efectiva que tiene un beneficio significativo sobre el dolor y la discapacidad en la osteoartritis de rodilla. ${ }^{70}$

Evidencia de alta calidad de 44 ensayos $(3,537$ participantes) indica que el ejercicio en osteoartritis redujo el dolor (diferencia de medias estandarizada $-0,49$; intervalo de confianza de $95 \%$ : -0.39 a -0.59 ) inmediatamente después del tratamiento. ${ }^{71}$

\section{Electroterapia/estimulación eléctrica neuromuscular}

Las Guías de OARSI mencionan que la evidencia fue mixta con respecto a la eficacia de los agentes físicos y las modalidades electroterapéuticas debido a la contradicción en los hallazgos, defectos de diseño 0 un bajo recuento de estudios similares. ${ }^{59}$

Zeng y colaboradores en 2015 concluyeron en una revisión sistemática y metaanálisis de 27 ensayos con 995 pacientes y seis tipos de electroestimulación (nerviosa eléctrica transcutánea de alta frecuencia, nerviosa eléctrica transcutánea de baja frecuencia, eléctrica neuromuscular, corriente interferencial, eléctrica pulsada y neuroestimulación interactiva no invasiva) que la corriente interferencial es el único tratamiento significativamente eficaz en cuanto a la intensidad del dolor y cambio en la puntuación del dolor en comparación con el grupo de control. ${ }^{72}$

\section{Uso del ultrasonido}

En una revisión sistemática y metaanálisis que incluyó 12 ensayos clínicos, se concluye que el ultrasonido terapéutico pulsado es más efectivo para aliviar el dolor y mejorar la función en osteoartritis que el ultrasonido continuo y el grupo control. ${ }^{73}$

\section{Terapia manual}

En una revisión sistemática y metaanálisis con 14 ensayos y 841 participantes con osteoartritis de rodilla, con pobre calidad metodológica y alta heterogeneidad entre los estudios, se concluye que la terapia manual puede ser efectiva para disminuir dolor y rigidez e incrementar la funcionalidad. ${ }^{74}$

Una revisión sistemática y metaanálisis con 12 ensayos apoya el uso de TM frente a una serie de comparadores diferentes para mejorar la función de la rodilla. Hubo menor apoyo para la reducción del dolor, y no se puede respaldar el rendimiento funcional en este momento. ${ }^{75}$

La guía de AAOS concluye que la terapia manual no se puede recomendar ni a favor ni en contra en los casos con osteoartritis debido a la falta de estudios que examinen la mayoría de las técnicas de terapia manual. ${ }^{38}$ La Guía de OARSI no incluyó a la terapia manual debido a insuficiente evidencia disponible. ${ }^{59}$

\section{Discusión}

La osteoartritis u osteoartritis postraumática en el atleta, adulto joven, económicamente activo y con necesidad de trabajar, implica un gran reto para el médico. El manejo de dicha patología hoy en día consiste por un lado en la mejora de los síntomas de dolor y función, y por el otro lado en realizar estrategias que permitan detener 0 al menos retrasar su evolución hacia una osteoartritis generalizada, y en el caso específico del atleta debemos buscar la mayor funcionalidad posible de sus articulaciones durante su carrera deportiva, un pronto regreso a ella y sin afectar la calidad de vida en su etapa adulta. El haber realizado una revisión sistemática de estudios de alto nivel de evidencia permitirá dar al médico más herramientas quirúrgicas y no quirúrgicas que permitan enriquecer el manejo multimodal de la osteoartritis en el atleta y perpetuar así la carrera deportiva y calidad de vida en el atleta a su cargo.

\section{Conclusiones}

Sí existen tratamientos quirúrgicos y no quirúrgicos efectivos y seguros publicados entre 2013 y 2018 para el manejo de la osteoartritis aplicable al atleta. Es importante seguir investigando y realizando estudios de alto nivel de evidencia para el tratamiento específico de la osteoartritis en el atleta que nos permitan enriquecer el manejo multimodal de la osteoartritis. 
La principal limitación es que no todos los estudios están enfocados en atletas, por lo cual las interpretaciones deben ser tomadas como herramientas de tratamiento posible en este tipo de casos, ya que los datos son de grupos de jóvenes activos y en edad productiva.

\section{Referencias}

1. Villalobos FEJ, Izaguirre A, Almazán A, Cruz F, Pérez-Jímenez FX, Ibarra JC. Articular cartilage injuries in 1,309 knee arthroscopies, a public health problem in a developing country? Osteoarthritis and Cartilage. 2007; 15 (Suppl. 2): B102.

2. Aroen A, Løken S, Heir S, Alvik E, Ekeland A, Granlund OG, Engebretsen L. Articular cartilage lesions in 993 consecutive knee arthroscopies - Am J Sports Med. 2004; 32 (1): 211-215.

3. Hjelle K, Solheim E, Strand T, Muri R, Brittberg M. Articular cartilage defects in 1,000 knee arthroscopies. Arthroscopy. 2002; 18 (7): 730-734.

4. Kramer W, Hendricks KK, Wang J. Pathogenetic mechanisms of posttraumatic osteoarthritis: opportunities for early intervention. Int J Clin Exp Med. 2011; 4 (4): 285-298.

5. Linden B. Osteochondritis dissecans of the femoral condyles: a long-term follow-up study. JBJS. 1977; 59 (6): 769-776.

6. Peña AA, Fernández-López JC. Prevalence and risk factors in osteoarthritis. Reumatol Clin. 2007; 3 (Suppl 3): S6-S12.

7. Shekelle PG, Woolf SH, Eccles M, Grimshaw J. Clinical guidelines: developing guidelines. BMJ. 1999; 318 (7183): 593-596.

8. Ajuied A, Wong F, Smith C, Norris M, Earnshaw P, Back D, Davies $A$. Anterior cruciate ligament injury and radiologic progression of knee osteoarthritis: a systematic review and meta-analysis. Am J Sports Med. 2014; 42 (9): 2242-2252.

9. Van Ginckel A, Verdonk P, Witvrouw E. Cartilage adaptation after anterior cruciate ligament injury and reconstruction: implications for clinical management and research? A systematic review of longitudinal MRI studies. Osteoarthritis Cartilage. 2013; 21 (8): 1009-1024.

10. Tsoukas D, Fotopoulos V, Basdekis G, Makridis KG. No difference in osteoarthritis after surgical and non-surgical treatment of ACL-injured knees after 10 years. Knee Surg Sports Traumatol Arthrosc. 2016; 24 (9): 2953-2959.

11. Gomoll AH, Gillogly SD, Cole BJ, Farr J, Arnold R, Hussey $\mathrm{K}$, et al. Autologous chondrocyte implantation in the patella: a multicenter experience. Am J Sports Med. 2014; 42 (5): 1074-1081.

12. Monk P, Garfjeld RP, Palmer AJ, Bayliss L, Mafi R, Beard D, et al. The urgent need for evidence in arthroscopic meniscal surgery. Am J Sports Med. 2017; 45 (4): 965-973.

13. Beaufils P, Becker R, Kopf S, Englund M, Verdonk R, Ollivier $\mathrm{M}$, et al. Surgical management of degenerative meniscus lesions: the 2016 ESSKA meniscus consensus. Knee Surg Sports Traumatol Arthrosc. 2017; 25 (2): 335-346.

14. Chen HW, Liu GD, Wu LJ. Clinical and radiological outcomes following arthroscopic-assisted management of tibial plateau fractures: a systematic review. Knee Surg Sports Traumatol Arthrosc. 2015; 23 (12): 3464-3472.

15. Namdari S, Skelley N, Keener JD, Galatz LM, Yamaguchi K. What is the role of arthroscopic debridement for glenohumeral arthritis? A critical examination of the literature. Arthroscopy. 2013; 29 (8): 1392-1398.
16. Sayegh ET, Mascarenhas R, Chalmers PN, Cole BJ, Romeo $A A$, Verma NN. Surgical treatment options for glenohumeral arthritis in young patients: a systematic review and metaanalysis. Arthroscopy. 2015; 31 (6): 1156-1166.e8.

17. Bloch B, Srinivasan S, Mangwani J. Current concepts in the management $1 \mathrm{f}$ ankle osteoarthritis: a systematic review. J Foot Ankle Surg. 2015; 54 (5): 932-939.

18. Noailles T, Lopes R, Padiolleau G, Gouin F, Brilhault J. Non-anatomical or direct anatomical repair of chronic lateral instability of the ankle: a systematic review of the literature after at least 10 years of follow-up - Foot Ankle Surg. 2018; 24 (2): 80-85.

19. Ross KA, Murawski CD, Smyth NA, Zwiers R, Wiegerinck Jl, Van Bergen CJ, et al. Current concepts review: arthroscopic treatment of anterior ankle impingement. Foot Ankle Surg. 2017; 23 (1): 1-8.

20. Cadossi M, Buda RE, Ramponi L, Sambri A, Natali S, Giannini $\mathrm{S}$. Bone marrow-derived cells and biophysical stimulation for talar osteochondral lesions: a randomized controlled study. Foot Ankle Int. 2014; 35 (10): 981-987.

21. VanTienderen RJ, Dunn JC, Kusnezov N, Orr JD. Osteochondral allograft transfer for treatment of osteochondral lesions of the talus: a systematic review. Arthroscopy. 2017; 33 (1): 217-222.

22. Johnson P, Lee DK. Evidence-based rationale for ankle cartilage allograft replacement: a systematic review of clinical outcomes. J Foot Ankle Surg. 2015; 54 (5): 940-943.

23. Thomas T, Amouroux F, Vincent P. Intra-articular hyaluronic acid in the management of knee osteoarthritis: pharmacoeconomic study from the perspective of the national health insurance system. PLoS One. 2017; 12 (3): e0173683.

24. Sun SF, Hsu CW, Lin HS, Liou IH, Chen YH, Hung CL. Comparison of single intra-articular injection of novel hyaluronan (HYA-JOINT Plus) with Synvisc-One for knee osteoarthritis: a randomized, controlled, double-blind trial of efficacy and safety. J Bone Joint Surg Am. 2017; 99 (6): 462-471.

25. Kearey P, Popple AE, Warren J, Davis T, Bellamy N. LOBRAS Study Group. Improvement in condition-specific and generic quality of life outcomes in patients with knee osteoarthritis following single-injection Synvisc: results from the LOBRAS study. Curr Med Res Opin. 2017; 33 (3): 409-419.

26. O'Hanlon CE, Newberry SJ, Booth M, Grant S, Motala A, Maglione MA, et al. Hyaluronic acid injection therapy for osteoarthritis of the knee: concordant efficacy and conflicting serious adverse events in two systematic reviews. Syst Rev. 2016; 5 (1): 186.

27. Ricci M, Micheloni GM, Berti M, Perusi F, Sambugaro E, Vecchini $E$, et al. Clinical comparison of oral administration and viscosupplementation of hyaluronic acid $(\mathrm{HA})$ in early knee osteoarthritis. Musculoskelet Surg. 2017; 101 (1): 45-49.

28. Maheu E, Rannou F, Reginster JY. Efficacy and safety of hyaluronic acid in the management of osteoarthritis: evidence from real-life setting trials and surveys. Semin Arthritis Rheum. 2016; 45 (4 Suppl): S28-33.

29. Campbell KA, Erickson BJ, Saltzman BM, Mascarenhas R, Bach BR Jr, Cole BJ, et al. Is local viscosupplementation injection clinically superior to other therapies in the treatment of osteoarthritis of the knee: a systematic review of overlapping meta-analyses. Arthroscopy. 2015; 31 (10): 2036-2045.e14.

30. Trojian TH, Concoff AL, Joy SM, Hatzenbuehler JR, Saulsberry WJ, Coleman $\mathrm{Cl}$. AMSSM scientific statement concerning viscosupplementation injections for knee osteoarthritis: importance for individual patient outcomes. Br J Sports Med. 2016; 50 (2): 84-92. 
31. Monticone M, Frizziero A, Rovere G, Vittadini F, Uliano D, LA Bruna $S$, et al. Hyaluronic acid intra-articular injection and exercise therapy: effects on pain and disability in subjects affected by lower limb joints osteoarthritis. A systematic review by the Italian Society of Physical and Rehabilitation Medicine (SIMFER). Eur J Phys Rehabil Med. 2016; 52 (3): 389-399.

32. Filardo G, Di Matteo B, Di Martino A, Merli ML, Cenacchi A, Fornasari $\mathrm{P}$, et al. Platelet-rich plasma intra-articular knee injections show no superiority versus viscosupplementation: a randomized controlled trial. Am J Sports Med. 2015; 43 (7): 1575-1582.

33. Giarratana LS, Marelli BM, Crapanzano C, De Martinis SE, Gala L, Ferraro M, et al. A randomized double-blind clinical trial on the treatment of knee osteoarthritis: the efficacy of polynucleotides compared to standard hyaluronian viscosupplementation.- Knee. 2014; 21 (3): 661-668.

34. Conrozier T, Eymard F, Afif N, Balblanc JC, Legré-Boyer V, Chevalier X. Happyvisc Study Group. Safety and efficacy of intra-articular injections of a combination of hyaluronic acid and mannitol (HAnOX-M) in patients with symptomatic knee osteoarthritis: Results of a double-blind, controlled, multicenter, randomized trial. Knee. 2016; 23 (5): 842-848.

35. Henrotin Y, Chevalier X, Deberg M, Balblanc JC, Richette P, Mulleman D, et al. Osteoarthritis Group of French Society of Rheumatology. Early decrease of serum biomarkers of type II collagen degradation (Coll2-1) and joint inflammation (Coll2-1 NO2 ) by hyaluronic acid intra-articular injections in patients with knee osteoarthritis: a research study part of the Biovisco study. J Orthop Res. 2013; 31 (6): 901-907.

36. Filardo G, Di Matteo B, Tentoni F, Cavicchioli A, Di Martino A, Lo Presti $M$, et al. No effects of early viscosupplementation after arthroscopic partial meniscectomy: a randomized controlled trial. Am J Sports Med. 2016; 44 (12): 3119-3125.

37. Di Martino A, Tentoni F, Di Matteo B, Cavicchioli A, Lo Presti $\mathrm{M}$, Filardo $\mathrm{G}$, et al. Early viscosupplementation after anterior cruciate ligament reconstruction: a randomized controlled trial. Am J Sports Med. 2016; 44 (10): 2572-2578.

38. Jevsevar D, Donnelly P, Brown GA, Cummins DS. Viscosupplementation for osteoarthritis of the knee: a systematic review of the evidence. J Bone Joint Surg Am. 2015; 97 (24): 2047-2060.

39. Jevsevar DS. Treatment of osteoarthritis of the knee: evidencebased guideline, 2nd edition. J Am Acad Orthop Surg. 2013; 21 (9): 571-576.

40. Trigkilidas D, Anand A. The effectiveness of hyaluronic acid intra-articular injections in managing osteoarthritic knee pain. Ann R Coll Surg Engl. 2013; 95 (8): 545-551.

41. Altman RD, Schemitsch E, Bedi A. Assessment of clinical practice guideline methodology for the treatment of knee osteoarthritis with intra-articular hyaluronic acid. Semin Arthritis Rheum. 2015; 45 (2): 132-139.

42. Lamo-Espinosa JM, Mora G, Blanco JF, Granero-Moltó F, Nuñez-Córdoba JM, Sánchez-Echenique C, et al. Intra-articular injection of two different doses of autologous bone marrow mesenchymal stem cells versus hyaluronic acid in the treatment of knee osteoarthritis: multicenter randomized controlled clinical trial (phase I/II). J TransI Med. 2016; 14 (1): 246.

43. Shapiro SA, Kazmerchak SE, Heckman MG, Zubair AC, O'Connor MI. A prospective, single-blind, placebo-controlled trial of bone marrow aspirate concentrate for knee osteoarthritis. Am J Sports Med. 2017; 45 (1): 82-90.

44. Xia $P$, Wang $X$, Lin Q, Li X. Efficacy of mesenchymal stem cells injection for the management of knee osteoarthritis: a systematic review and meta-analysis. Int Orthop. 2015; 39 (12): 2363-2372.

45. Yubo M, Yanyan L, Li L, Tao S, Bo L, Lin C. Clinical efficacy and safety of mesenchymal stem cell transplantation for osteoarthritis treatment: a meta-analysis. PLoS One. 2017; 12 (4): e0175449.

46. Pas HI, Winters M, Haisma HJ, Koenis MJ, Tol JL, Moen MH. Stem cell injections in knee osteoarthritis: a systematic review of the literature. Br J Sports Med. 2017; 51 (15): 1125-1133.

47. Meheux CJ, McCulloch PC, Lintner DM, Varner KE, Harris JD. Efficacy of intra-articular platelet-rich plasma injections in knee osteoarthritis: a systematic review. Arthroscopy. 2016; 32 (3): 495-505.

48. Dai WL, Zhou AG, Zhang H, Zhang J. Efficacy of platelet-rich plasma in the treatment of knee osteoarthritis: a meta-analysis of randomized controlled trials. Arthroscopy. 2017; 33 (3): 659670.e1.

49. Laudy AB, Bakker EW, Rekers M, Moen MH. Efficacy of platelet-rich plasma injections in osteoarthritis of the knee: a systematic review and meta-analysis. Br J Sports Med. 2015; 49 (10): 657-672.

50. Smith PA. Intra-articular autologous conditioned plasma injections provide safe and efficacious treatment for knee osteoarthritis: an FDA-sanctioned, randomized, double-blind, placebo-controlled clinical trial. Am J Sports Med. 2016; 44 (4): 884-891.

51. Simental-Mendía M, Vílchez-Cavazos JF, Peña-Martínez VM, Said-Fernández S, Lara-Arias J, Martínez-Rodríguez HG. Leukocyte-poor platelet-rich plasma is more effective than the conventional therapy with acetaminophen for the treatment of early knee osteoarthritis. Arch Orthop Trauma Surg. 2016; 136 (12): 1723-1732.

52. Gormeli G, Gormeli CA, Ataoglu B, Colak C, Aslantürk O, Ertem K. Multiple PRP injections are more effective than single injections and hyaluronic acid in knees with early osteoarthritis: a randomized, double-blind, placebo-controlled trial. Knee Surg Sports Traumatol Arthrosc. 2017; 25 (3): 958-965.

53. Gobbi A, Lad D, Karnatzikos G. The effects of repeated intra-articular PRP injections on clinical outcomes of early osteoarthritis of the knee. Knee Surg Sports Traumatol Arthrosc. 2015; 23 (8): 2170-2177.

54. Cole BJ, Karas V, Hussey K, Pilz K, Fortier LA. Hyaluronic acid versus platelet-rich plasma: a prospective, double-blind randomized controlled trial comparing clinical outcomes and effects on intra-articular biology for the treatment of knee osteoarthritis. Am J Sports Med. 2017; 45 (2): 339-346.

55. Zeng C, Wei J, Li H, Wang YL, Xie DX, Yang T, et al. Effectiveness and safety of Glucosamine, chondroitin, the two in combination, or celecoxib in the treatment of osteoarthritis of the knee. - Sci Rep. 2015; 5: 16827.

56. Kanzaki N, Ono Y, Shibata H, Moritani T. Glucosaminecontaining supplement improves locomotor functions in subjects with knee pain: a randomized, double-blind, placebocontrolled study. Clin Interv Aging. 2015; 10: 1743-1753.

57. Kongtharvonskul J, Anothaisintawee T, McEvoy M, Attia $\mathrm{J}$, Woratanarat $\mathrm{P}$, Thakkinstian A. Efficacy and safety of glucosamine, diacerein, and NSAIDs in osteoarthritis knee: a systematic review and network meta-analysis. Eur J Med Res. 2015; 20: 24.

58. Bruyere O, Cooper C, Pelletier JP, Branco J, Brandi $\mathrm{ML}$, Guillemin $\mathrm{F}$, et al. An algorithm recommendation for the management of knee osteoarthritis in Europe and internationally: a report from a task force of the European 
Society for Clinical and Economic Aspects of Osteoporosis and Osteoarthritis (ESCEO). Semin Arthritis Rheum. 2014; 44 (3): 253-263.

59. McAlindon TE, Bannuru RR, Sullivan MC, Arden NK, Berenbaum F, Bierma-Zeinstra SM, et al. OARSI guidelines for the non-surgical management of knee osteoarthritis. Osteoarthritis Cartilage. 2014; 22 (3): 363-388.

60. Kongtharvonskul J, Woratanarat P, McEvoy M, Attia J, Wongsak S, Kawinwonggowit V, Thakkinstian A. Efficacy of glucosamine plus diacerein versus monotherapy of glucosamine: a double-blind, parallel randomized clinical trial. Arthritis Res Ther. 2016; 18 (1): 233.

61. Kwoh CK, Roemer FW, Hannon MJ, Moore CE, Jakicic JM, Guermazi A, et al. Effect of oral glucosamine on joint structure in individuals with chronic knee pain: a randomized, placebocontrolled clinical trial. Arthritis Rheumatol. 2014; 66 (4): 930-939.

62. Liu X, Machado GC, Eyles JP, Ravi V, Hunter DJ. Dietary supplements for treating osteoarthritis: a systematic review and meta-analysis. Br J Sports Med. 2018; 52 (3): 167-175.

63. Jüni P, Hari R, Rutjes AW, Fischer R, Silletta MG, Reichenbach $\mathrm{S}$, et al. Intra-articular corticosteroid for knee osteoarthritis. Cochrane Database Syst Rev. 2015; (10): CD005328.

64. Soriano-Maldonado A, Klokker L, Bartholdy C, Bandak E, Ellegaard K, Bliddal H, et al. Intra-articular corticosteroids in addition to exercise for reducing pain sensitivity in knee osteoarthritis: exploratory outcome from a randomized controlled trial. PLoS One. 2016; 11 (2): e0149168.

65. Popma JW, Snel FW, Haagsma CJ, Brummelhuis-Visser P, Oldenhof HG, van der Palen J, et al. Comparison of 2 dosages of intraarticular triamcinolone for the treatment of knee arthritis: results of a 12-week randomized controlled clinical trial. J Rheumatol. 2015; 42 (10): 1865-1868.

66. McAlindon TE, LaValley MP, Harvey WF, Price LL, Driban $\mathrm{JB}$, Zhang $\mathrm{M}$, et al. Effect of intra-articular triamcinolone vs saline on knee cartilage volume and pain in patients with knee osteoarthritis: a randomized clinical trial. - JAMA. 2017; 317 (19): 1967-1975.

67. Osteoarthritis: Care and Management in Adults. National Clinical Guideline Centre (UK) London: National Institute for
Health and Care Excellence (UK); 2014? Feb. National Institute for Health and Clinical Excellence: Guidance.

68. Uthman OA, Van derWindt DA, Jordan JL. Exercise for lower limb osteoarthritis: systematic review incorporating trial sequential analysis and network meta-analysis. BMJ. 2013; 347: 1-13.

69. Hall M, Hinman RS, Van Der Esch M. Is the relationship between increased knee muscle strength and improved physical function following exercise dependent on baseline physical function status? Arthritis Res Ther. 2017; 19: 271.

70. Coudeyre E, Jegu AG, Giustanini M. Isokinetic muscle strengthening for knee osteoarthritis: A systematic review of randomized controlled trials with meta-analysis. Ann Phys Rehabil Med. 2016; 59: 207-215.

71. Fransen M, McConnell S., Exercise for osteoarthritis of the knee. Cochrane Database Syst Rev. 2015; 1: CD004376.

72. Zeng $\mathrm{C}$, Li H, Yang T, Deng ZH. Electrical stimulation for pain relief in knee osteoarthritis: systematic review and network meta-analysis. Osteoarthritis Cartilage. 2015; 23 (2): 189-202

73. Zeng C, Li H, Yang T, Deng ZH. Effectiveness of continuous and pulsed ultrasound for the management of knee osteoarthritis: a systematic review and network meta-analysis. Osteoarthritis Cartilage. 2014; 22 (8): 1090-1099.

74. Xu Q, Chen B, Wang Y, Wang $X$. The effectiveness of manual therapy for relieving pain, stiffness, and dysfunction in knee osteoarthritis: a systematic review and meta-analysis. Pain Physician. 2017; 20 (4): 229-243.

75. Salamh P, Cook C, Reiman MP, Sheets C, Treatment effectiveness and fidelity of manual therapy to the knee: a systematic review and meta-analysis. Musculoskeletal Care. 2017; 15 (3): 238-248.

\section{Fuente de financiamiento}

El presente estudio contó con el apoyo académico irrestricto de Sanofi. 